\title{
Strain-Induced Bandgap Enhancement of InSe Ultrathin Films with Self-Formed Two-Dimensional Electron Gas
}

Zhimo Zhang ${ }^{1}$, Yuan Yuan ${ }^{1}$, Weiqing Zhou ${ }^{2}$, Chen Chen $^{3,4}$, Shengjun Yuan ${ }^{2}$, Hualing Zeng ${ }^{3,4}$,

$$
\text { Ying-Shuang } \mathrm{Fu}^{1,{ }^{*}} \text {, Wenhao Zhang }{ }^{1, \#}
$$

1. School of Physics and Wuhan National High Magnetic Field Center, Huazhong University of Science and Technology, Wuhan 430074, China

2. Key Laboratory of Artificial Micro- and Nano-structures of Ministry of Education and School of Physics and Technology, Wuhan University, Wuhan 430072, China

3. International Center for Quantum Design of Functional Materials (ICQD), Hefei National Laboratory for Physical Science at the Microscale, and Synergetic Innovation Center of Quantum Information and Quantum Physics, University of Science and Technology of China, Hefei, Anhui 230026, China

4. Key Laboratory of Strongly-Coupled Quantum Matter Physics, Chinese Academy of Sciences, Department of Physics, University of Science and Technology of China, Hefei, Anhui 230026, China

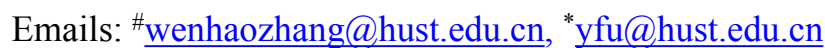




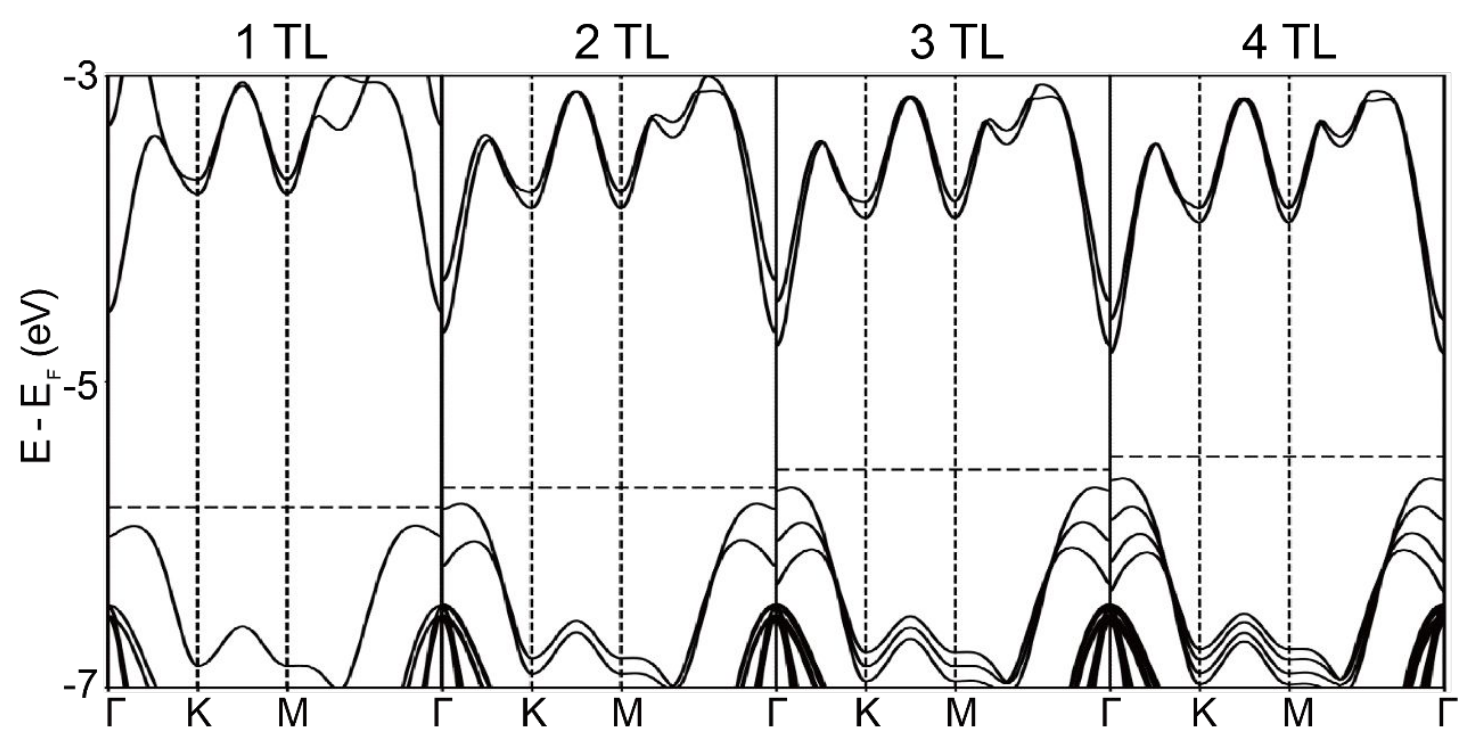

Figure S1. Layer-dependent band structure for 1-4 TL InSe. Vacuum level $\mathrm{E}_{\mathrm{vac}}$ is set to $0 \mathrm{eV}$, and $\mathrm{E}_{\mathrm{vac}}-\mathrm{E}_{\mathrm{F}}=\mathrm{E}_{\mathrm{wf}}$, where $\mathrm{E}_{\mathrm{F}}$ is the Fermi level and $\mathrm{E}_{\mathrm{wf}}$ is the work function. 


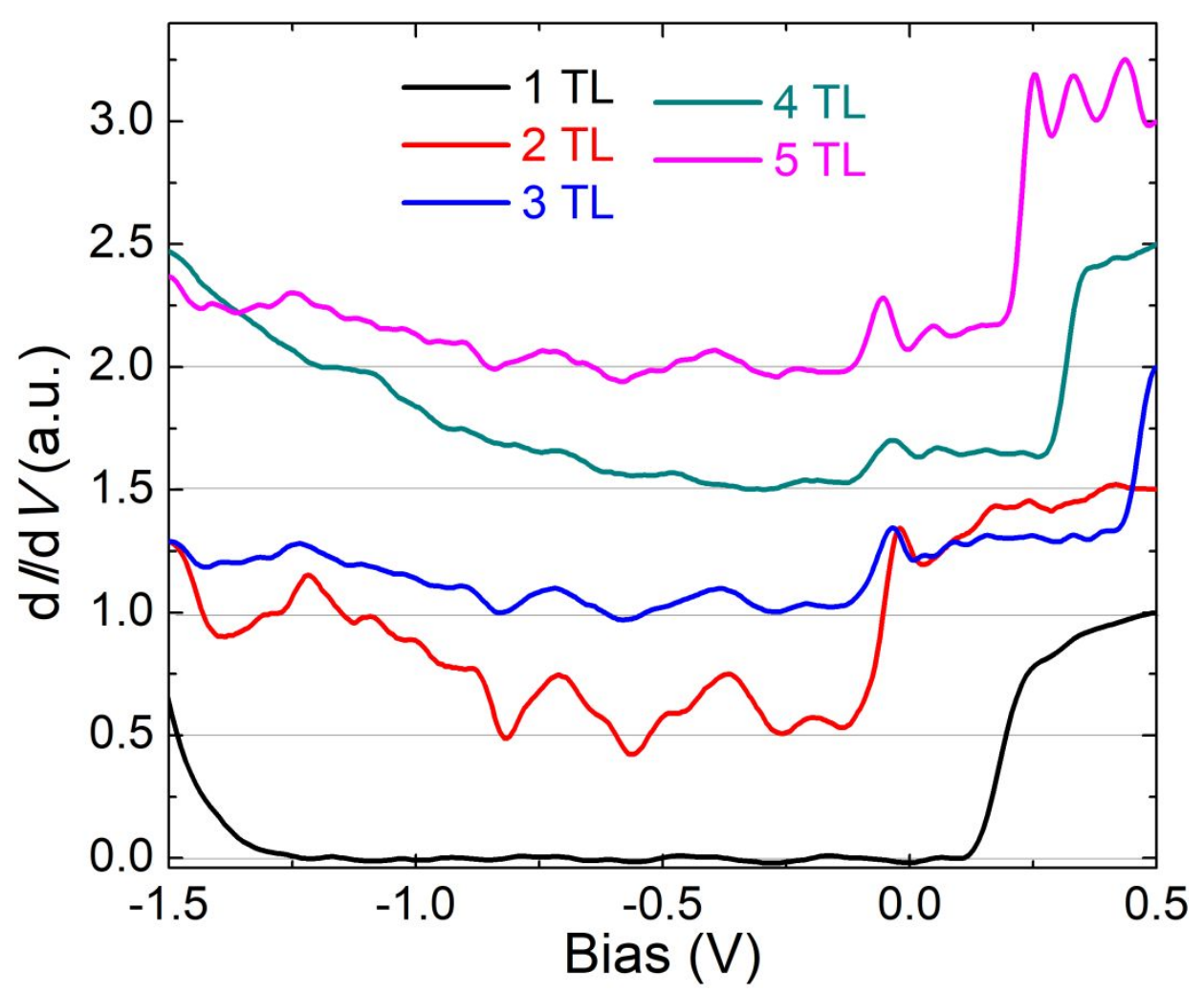

Figure S2. A close-up of STS spectra in a linear scale for Fig. 3(a) in a narrowed energy range, showing obvious states of nonvanishing conductance inside the band gaps except for $1 \mathrm{TL}$. Spectra are shifted vertically with the horizontal gray line marking the zero differential conductance for each curve. 

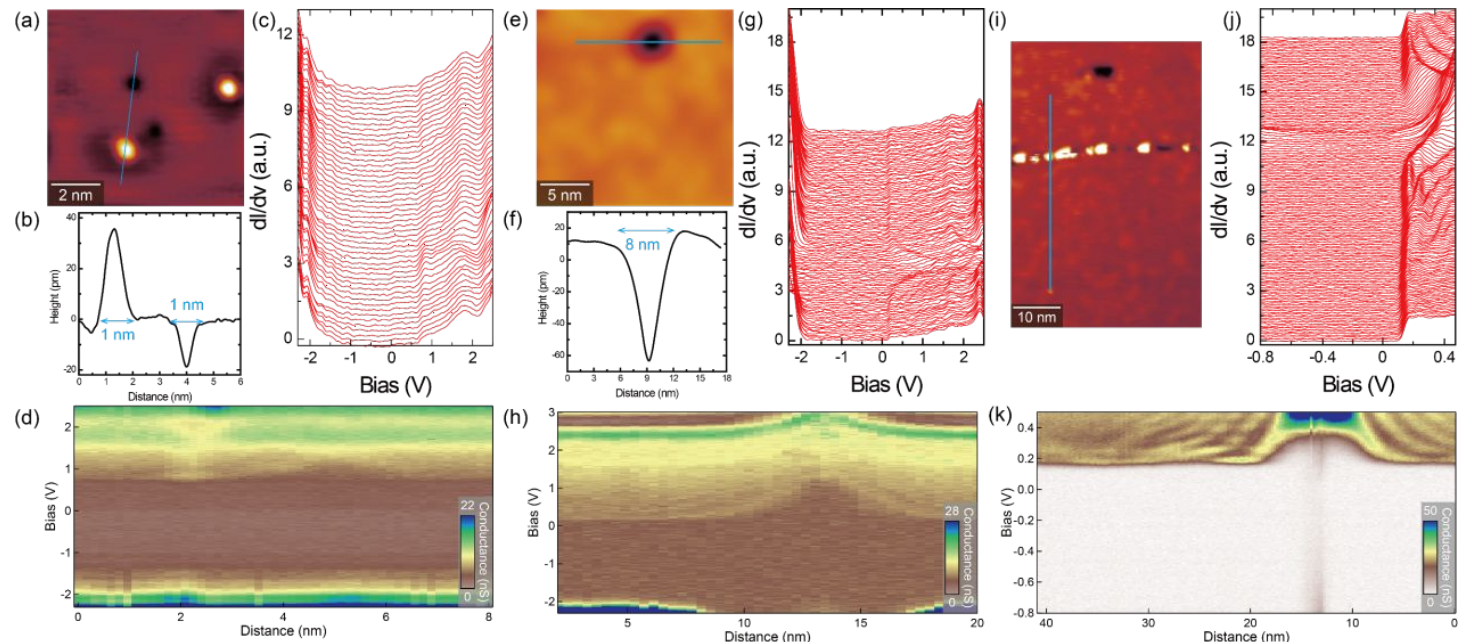

Figure S3 (a) STM image of 1 TL InSe film containing light defect with both bright protrusion and dark hole. (b) Line profile across the defects along the cyan line in (a). The light defect is restricted within $\sim 1 \mathrm{~nm}$ width and 20-40 pm depth. (c) Series of tunneling spectra measured along the cyan line in (a), indicating that the spectra are rather homogenous even though the surface is disordered. (d) 2D plot of (c). (e)-(h) The same as (a)-(d) but for an extended defect, which is deeper and larger with spatial extension enlarged to $\sim 8-10 \mathrm{~nm}$ width and $80 \mathrm{pm}$ depth. The affected STS also extends to $\sim 8-10 \mathrm{~nm}$ width. Obvious band bending is observed across the defect. (i)-(k) Similar to (a)-(d) but for both light and extended defects. Clearly, no connection can be built between the defects and the mid-gap states. 
(a)

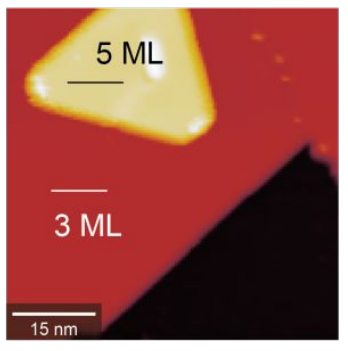

(b)

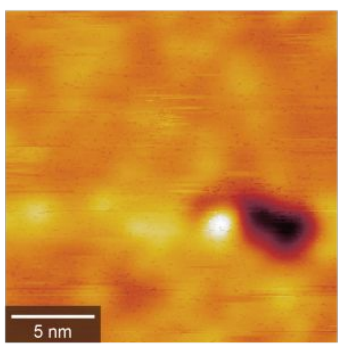

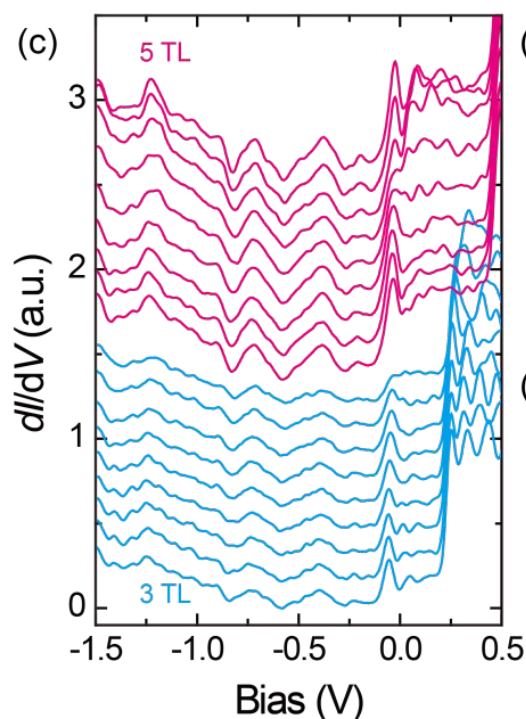
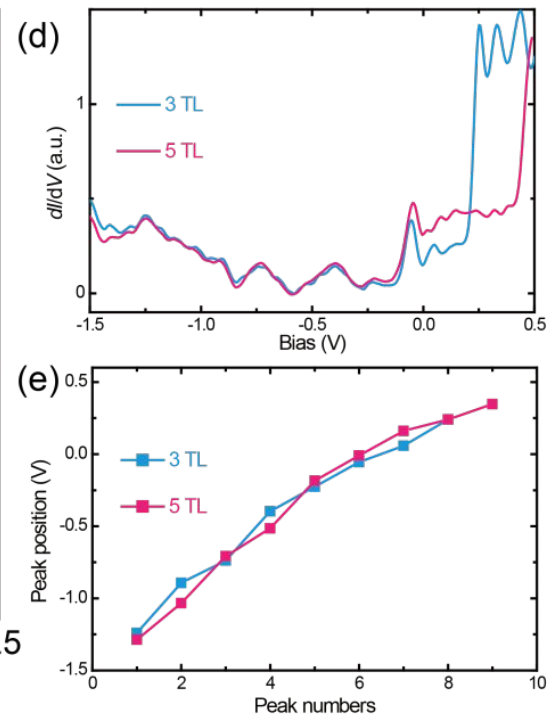

Figure S4. (a) Typical STM images for InSe surface containing both 3 TL and 5 TL terraces $(60 \times$ $60 \mathrm{~nm}^{2}, \mathrm{~V}_{\text {bias }}=+1.5 \mathrm{~V}, \mathrm{I}_{\mathrm{t}}=10 \mathrm{pA}$ ). (b) The zoom-in STM image of $3 \mathrm{TL} \mathrm{In}_{2} \mathrm{Se}_{3}$, showing a disordered surface $\left(20 \times 20 \mathrm{~nm}^{2}, V_{\text {bias }}=+0.1 \mathrm{~V}, \mathrm{I}_{\mathrm{t}}=50 \mathrm{pA}\right)$. (c) Series of tunneling spectra measured along the white and black lines in (a) and (b), respectively. Even though the surface is disordered, both spectra are rather homogenous. (d) Point $\mathrm{d} I / \mathrm{d} V$ spectra on 3 and $5 \mathrm{TL}$ are compared by adjusting the intensities into similar levels, where the positions of peaks nearly coincide. (e) Peak evolutions of in-gap states for 3 and $5 \mathrm{TL}$, with a $\sim 0.2 \mathrm{~V}$ upwards shift for energies of $5 \mathrm{TL}$. Both give similar linear relationship with coincident slop, indicating uniform spacing interval of in-gap states. From this point of view, we trend to ascribe the in-gap states as energy subbands of quantized states. The shift of energy may possibly be ascribed to the inhomogeneous chemical potential or thicknessdependent quantum-confined states for different TL. However, not only the number of peaks, but also their energy intervals vary a lot from layer to layer, as well as for different samples. This is inconsistent with the scenario of quantum well states. 

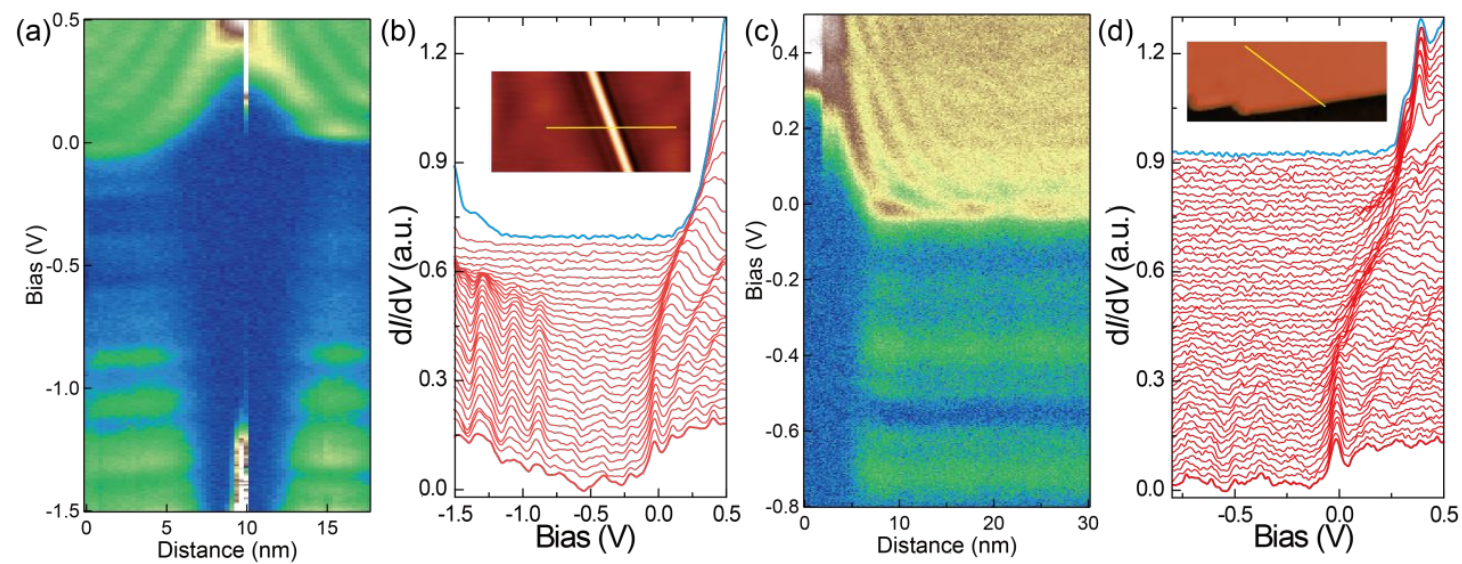

Figure S5 (a) 2D plot of STS spectra across the defect along the yellow line in inset of (b). (b)

Selected of tunneling spectra along the line. The in-gap states are strongly suppressed at the extended defect labeled by cyan. (c),(d) Similar to (a),(b) but for a step edge. We find the in-gap states are strongly suppressed in the band bending case, as shown by the cyan curves. Concomitantly, the intensities of in-gap states share similar modulations as the CB edges, which result from the standing waves. These indicate an inherent origin of InSe itself rather than the external factors. 

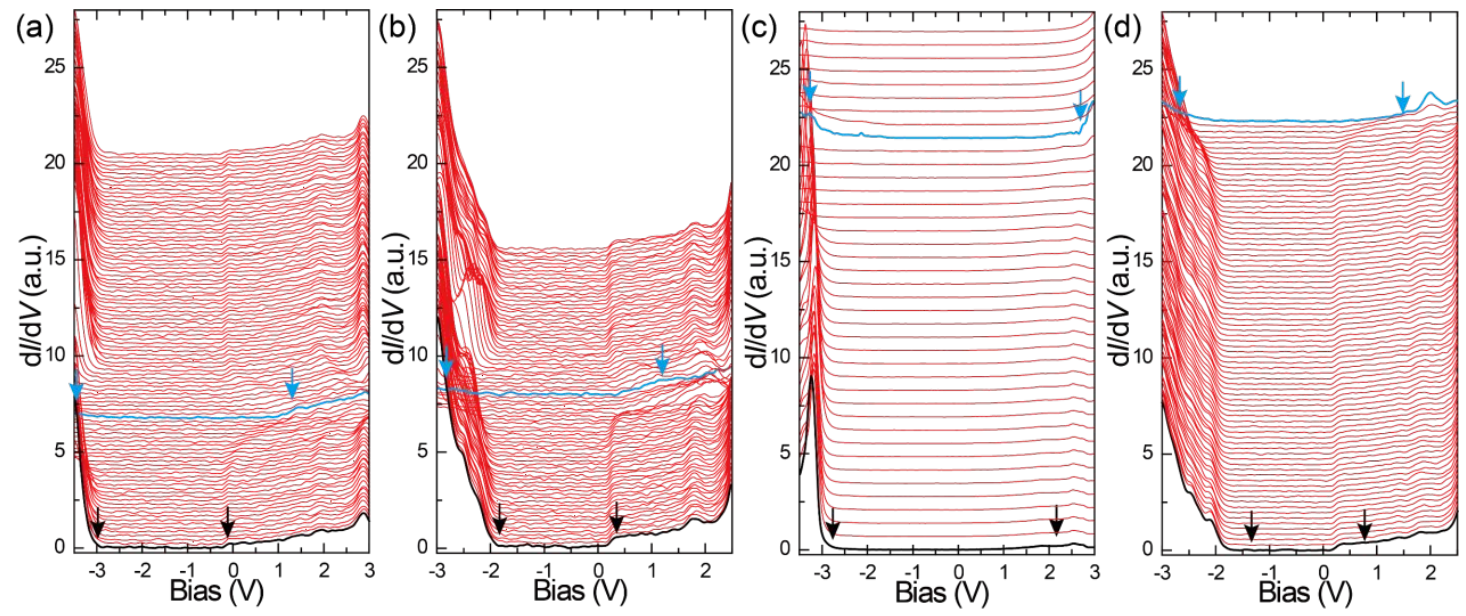

Figure S6. Series of tunneling spectra across various defects/step boundaries, the same as the 2D plot of Figs. 4(a')-(d'). The black and cyan curves donate the $\mathrm{dI} / \mathrm{dV}$ taken far away and right at the defects/step boundaries, respectively. Arrows label the more visible shoulders of CB/VB branches. To avoid missing the deviation of CB/VB branches, we measure the STS linecuts over a very wide energy range $( \pm 3 \mathrm{~V})$. However, in the $2 \mathrm{D}$ plots of tunneling spectra of Fig. 4 , we find it not apparently convenient to trace the onsets of $\mathrm{CB} / \mathrm{VB}$ edges because of the poor contrast of conductance. Instead, we trace the shoulders of the $\mathrm{CB} / \mathrm{VB}$ edges to highlight the evolution of band gap when approaching the defects/step boundaries. Fig. R5 illustrates the differences. As a result, the gap size is globally larger than the actually gap in Fig. 3. However, this does not influence the trends of the band bending behaviors. 

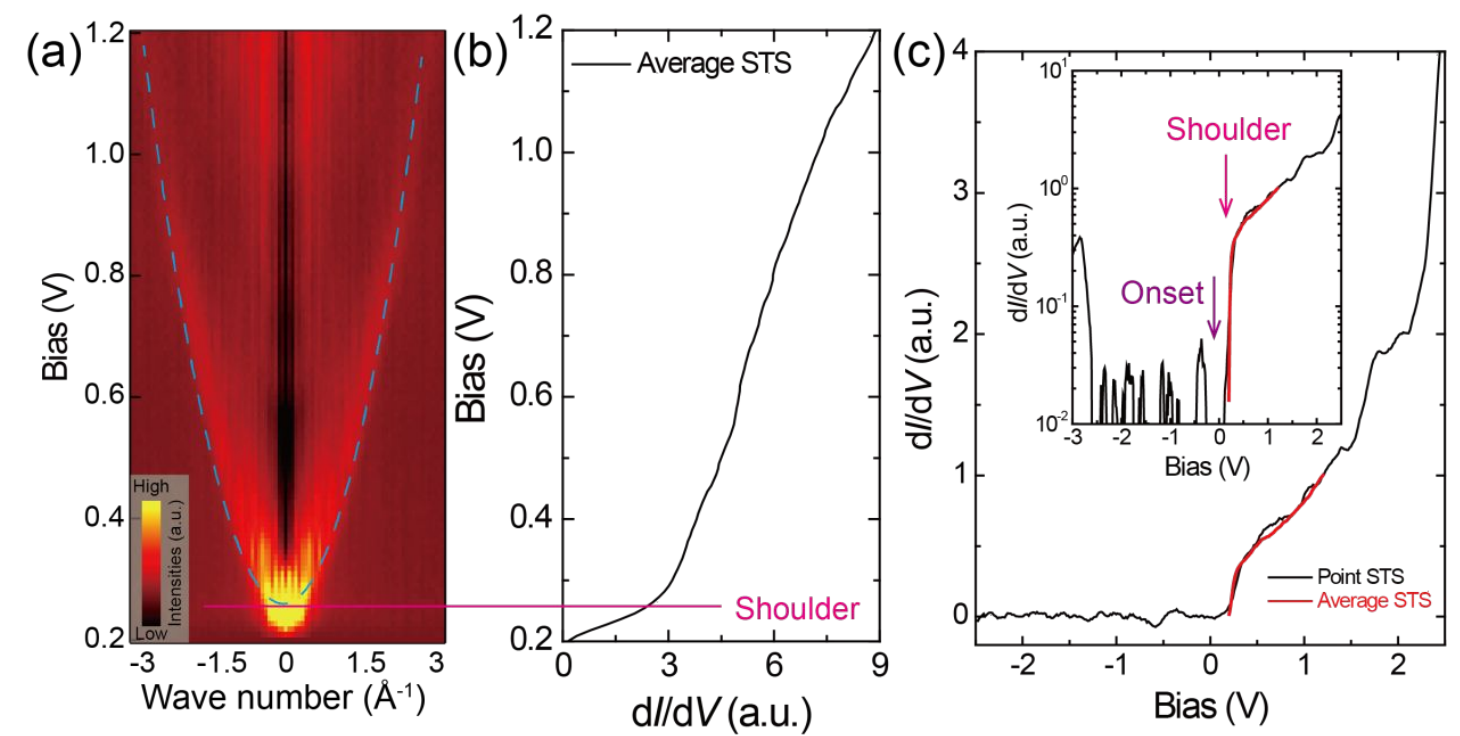

Figure S7 Comparison for the QPI observations (a) and the averaged STS (c) in the same energy scale. (c) Comparison for the averaged and single point STS. Inset is plotted in a logarithmic scale marked with the onset and shoulder of CB. The strongest intensities of CBM extracted from QPI observations is $\sim 0.25 \mathrm{~V}$, which merely corresponds to the shoulder of the CB branch in the STS spectrum, whereas the onset is far below $0.2 \mathrm{~V}$. We also compare the averaged and single point STS in a logarithmic scale, labeling with the onset and shoulder of CB. Such discrepancy basically stems from the nonnegligible conductance for observations with visible intensities, whereas the signal of the onset of $\mathrm{d} I / \mathrm{d} V$ is too weak to contribute the QPI patterns. So, the gap determination from the QPI measurements seems not a suitable method for quantification. Nevertheless, the energy difference between the onset and shoulder of $\mathrm{CB} / \mathrm{VB}$ is $\sim 0.3 \mathrm{eV}$, giving rise to an enlargement of $\sim 0.6 \mathrm{eV}$ for the band gap size. This is also consistent with the difference between point STS spectra (Fig. 3) and 2D plots (Fig. 4). 

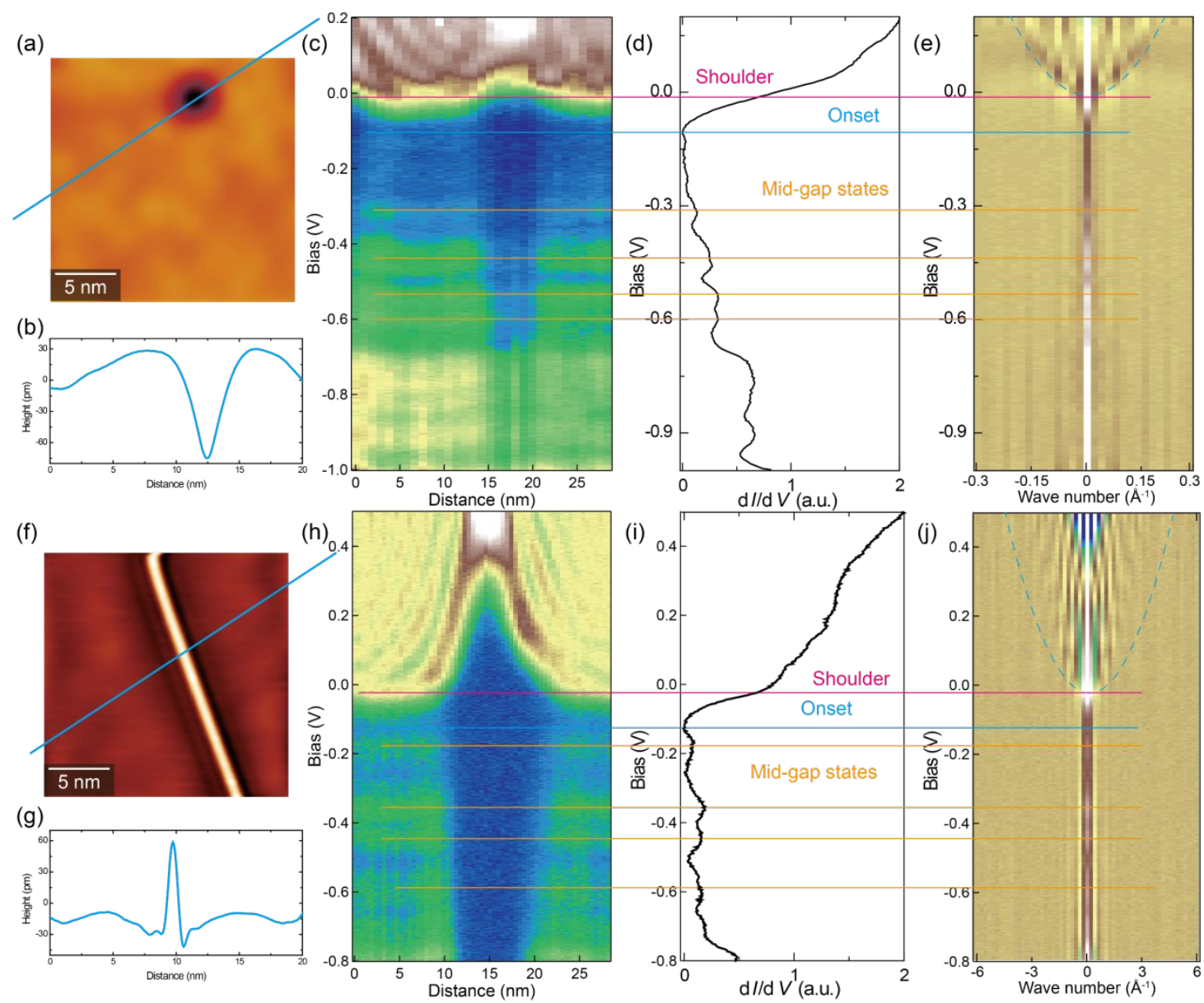

Figure S8 (a),(b) STM image and line profile of an extended defect (9 nm width and $100 \mathrm{pm}$ depth) on a 2 TL InSe film. (c) 2D plot of STS spectra across the defect along the cyan line in (a). (d) Averaged STS of (c) in the same energy scale. (e) FFT plot of (b), showing the energy dispersion. (f)-(j) Similar to (a)-(d) but on a ridge defect (3 $\mathrm{nm}$ width and $100 \mathrm{pm}$ depth). 
(a)
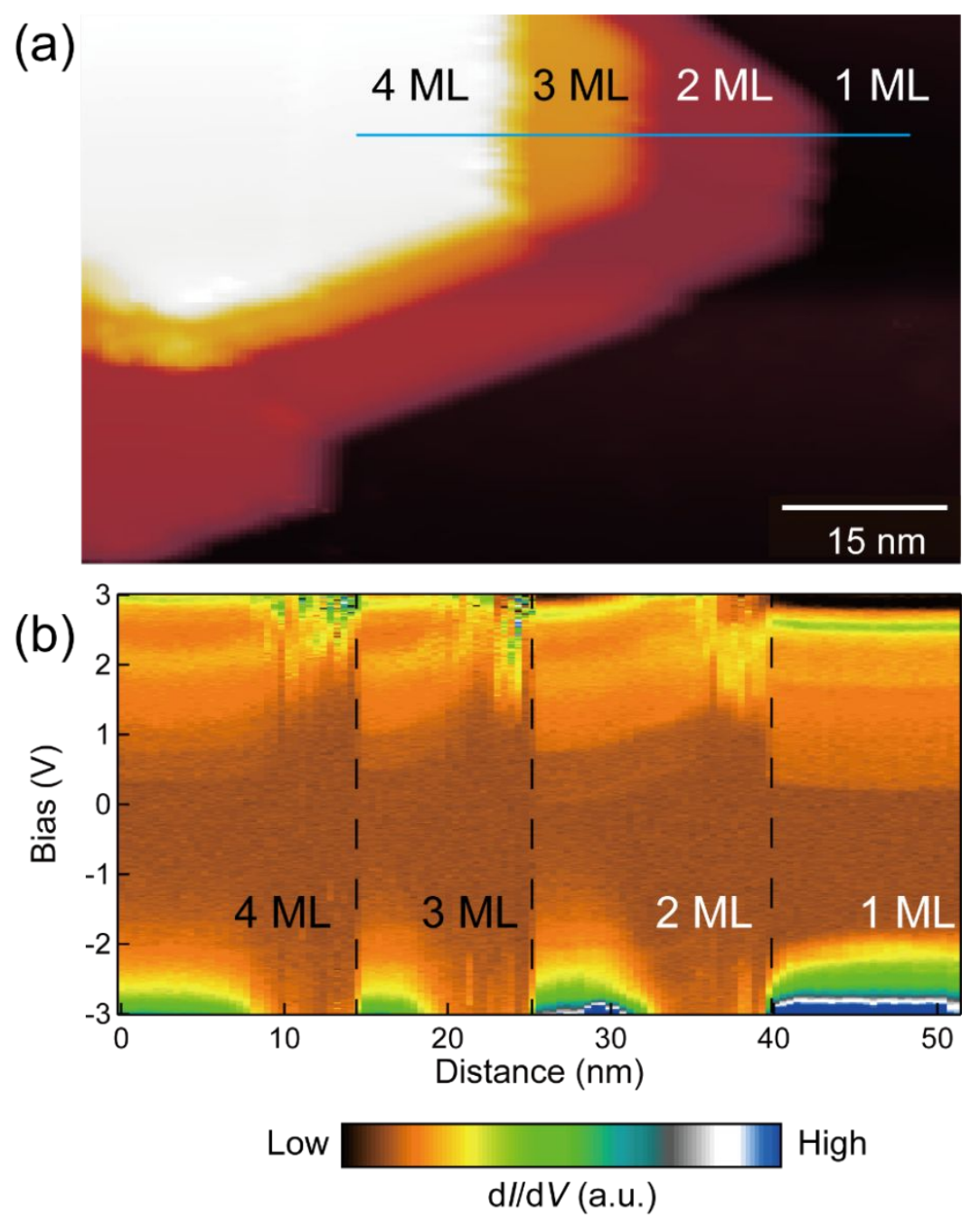

Figure S9. (a) Typical STM images for InSe surface containing 1-4 TL terraces $\left(80 \times 50 \mathrm{~nm}^{2}, \mathrm{~V}_{\text {bias }}\right.$ $=+3.0 \mathrm{~V}, \mathrm{I}_{\mathrm{t}}=10 \mathrm{pA}$ ). (b) $2 \mathrm{D}$ plot of tunneling spectra measured along the cyan line in (a). Vertical dashed lines mark the positions of step edges. Obviously, the band bending of upward for CB and downward for VB occur mainly on the upper terrace of the step, while the spectra on lower terrace show little bending features. 